\title{
RETRACTED ARTICLE: Effects of Common Polymorphisms in the MTHFR and $A C E$ Genes on Diabetic Peripheral Neuropathy Progression: a Meta-Analysis
}

\author{
Shuai Wu • Yan Han • Qiang Hu • Xiao-Jie Zhang • \\ Guang-Cheng Cui $\cdot$ Ze-Zhi Li • Yang-tai Guan
}

Received: 18 September 2014 / Accepted: 30 October 2014 / Published online: 17 August 2015

(C) Springer Science+Business Media New York 2014

The Publisher and Editor retract this article in accordance with the recommendations of the Committee on Publication Ethics (COPE). After a thorough investigation we have strong reason to believe that the peer review process was compromised.

The original article was published online on November 25, 2014.

S. Wu $\cdot$ Y. Han · Z.-Z. Li $(\bowtie) \cdot$ Y.-t. Guan $(\bowtie)$

Department of Neurology, Shanghai Changhai Hospital, Secondary

Military Medical University, No. 168 Changhai Road,

Shanghai 200433, China

e-mail: lizezhi1981@aliyun.com

e-mail: yangtaiguan@hotmail.com

Q. Hu

Department of Psychology, Qiqihar Mental Health Center,

Qiqihar 161000, China

\section{X.-J. Zhang}

Department of Pathology, Qiqihar Medical University,

Qiqihar 161000, China

G.-C. Cui

Mental Health Institute, Qiqihar Medical University, Qiqihar 161000,

China 